\title{
Hybrid Precoding/Combining for single-user and Multi-Usersin mm-Wave MIMO systems
}

\author{
Nazeerunnisa, Madhavi Tatineni
}

\begin{abstract}
Wave is one of the potential $5 G$ technology predominant at high frequency. The antenna arrays allows parallel transmission to the multiple users. Due to the constraints in hardware in mm-Wave systems,it is difficult to implement conventional multiuser MIMO precoding techniques at mm-Wave. In this paper initially Hybrid mm-Wave MIMO capacity is compared with conventional MIMOperformance, the simulation results demonstrates that the performance loss is due to assumption of the quantized angle of arrival and departures in dictionary which is very small using OMP algorithm and the capacity approaches the conventional MIMO performance. Then the Hybrid Precoding (HP)at mm-Wave is formulated as a sparse optimization problem in which the hybrid MIMO precoders and combiners are used as the measurement matrices.Here transmit precoding and combining at the receiver is considered with a limited feedback as knowledge of channel may not be practically available.Orthogonal matching Pursuit(OMP) algorithm is used for single-user case and alsoin Multi-user case using simultaneous Orthogonal matching pursuit(SOMP) algorithm. In single user case numerical results are presented which shows that the proposed algorithm approach is closer to their unconstraint performance even after considering hardware constraints. Multi-user precoding results illustrates that the proposed $\mathrm{HP}$ method offers higher Spectral efficiency (SE) compared to analog -only beamforming $(\mathrm{AB})$ and is close tothe performance bench mark of optimal digital beamformer .
\end{abstract}

Keywords- mm-Wave, Hybrid Precoding/combining, multi-user, MIMO, Orthogonal Matching Pursuit

\section{INTRODUCTION}

$\mathrm{M}$ llimeter wave(mm-Wave) technology is emerging as the key holder towards achieving higher data rates [1], [2] in $5 \mathrm{G}$ networks, which have the potential to enable the different applications such as wireless $\mathrm{HD}, \mathrm{V} 2 \mathrm{X}$ (vehicle to everything) and virtual reality communication. However, the practical realization of $\mathrm{mm}-$ Wave technology faces several challenges, such as higher path losses [3], increased hardware complexity [1], [4] and severe signal blockage [3]. This has led researchers to explore the practical solutions which addresses these challenges, without significantly affecting the overall performance of the system. In order to overcome the path-loss in mm-Wave system, highly directional beamforming is required at transmitter $(\mathrm{Tx})$ and receiver $(\mathrm{Rx})$ [5]. Large antennas should be employed at base station (BS) and mobile station (MS) in mm-Wave to achieve high quality

Revised Manuscript Received on December 13, 2019.

* Correspondence Author

Nazeerunnisa*, MuffakhamJah College of Engineering and technology Hyderabad, Telangana, India.Email:nazeerqureshey13@gmail.com

Dr. MadhaviTatineni,GITAM, (Deemed to be university),Vizag, Andhrapradesh, India. Email: madhavi.tatineni@gitam.edu communication[6]. The BS need to serve parallely multiple users with different streams of data, which needs some precoding technique to be applied to generate the signal to be transmitted at the BS. In [7]-[11] proposes analog beamforming for a single user mm-Wave system which controls the angle of the transmitted signal at every antenna through a network of phase shifters,implemented in RF domain. It was a part of IEEE 802.11 ad [12], IEEE 802.15,3c [13] which is a commercial mm-Wave indoor communication standard. A joint design of analog beamforming vectors by the Tx and Rx using adaptive algorithms for beamforming and also code books with multi resolution were developed [7], [8]. To support multiple streams of data and more efficient beamforming,[6][14][15] proposed HP technique.Thanks to the inherent sparse nature of mm-Wave channels, a low complexity algorithm [6] using the concept of basis pursuit was developed for HP, with assumption of the knowledge of channel. In [14] a hybrid beamforming algorithm with the objective of maximizing the sum-rate or the received signal strength over different subcarriers were proposed for single user MIMO-OFDM system.In [15] HP algorithm that need partial channel knowledge of mm-Wave were developed. The main problem of above mentioned approaches is that it can support only limited number of streams. In multiuser systems, the digital precoding part of the HP gives more freedom in precoder design compared to analog only solutions, which can be exploited to minimize interference between multiple users. Hence designing and developing a low-complexity HP algorithms for multiple user mm-Wave systems is of significant research interest.

In this paper, analysis of HP for single user(SU) and multiple users(MU)based on OMP algorithm is presented and compared with analog only beamforming and with bench mark optimal digital precoding solution.

The paper is structured in the subsequent sections as follows: Section II describes the mm-Wave Hybrid MIMO system Model for MU, Section III describes the Hybrid precoding/combining(HPC) design problemformulation for SU using OMP, Section IV describes the problem formulation in mm-Wave Hybrid MIMO precoding/combining for MUand Section Vpresents results using MATLAB, which demonstrates the advantage of the proposed hybrid algorithm over $\mathrm{AB}$ andHP performance approaching Optimal digital precoder. Last Section VI holds the conclusion with future work. 


\section{Hybrid Precoding/Combining for single-user and Multi-Usersin mm-Wave MIMO Systems}

\section{Notations:}

Lower case $z$ represent a vector while the upper case $Z$ represents a matrix. The given superscripts $Z^{T}, Z^{*}, Z^{-1}, Z^{H}$ denotes the transpose, the conjugate, the inverse, the Hermitian (conjugate-transpose) of the matrix $Z$ respectively.

\section{MM-WAVE MULTI-USER HYBRID MIMO SYSTEM MODEL}

Consider a MUmm-Wave system model as shown in Fig.1 A BS with $\left(N_{B S}\right)$ transmitting antennas and RF chains $\left(N_{R F}\right)$ is assume to communicate with $\mathrm{M}$ mobile stations(MS) and these MS's are connected with a limited feedback to BS. Each MS has $\left(N_{M S}\right)$ antennas asshown in Fig.2. The aim is on representing the mm-Wave MUhybrid MIMObeamforming system model where the BS communicates every MS via only one single stream,hence the total count of streams $N_{s}=\mathrm{M}$.Also assuming that maximum users that can be served parallel by the BS is equal to $N_{R F}$ i.e $\mathrm{M} \leq N_{R F}$.

In Downlink the BS applies a $\mathrm{M}$ x $\mathrm{M}$ base-band precoder $F_{B}=\left[f_{1}^{B}, f_{2}^{B} \ldots \ldots f_{M}^{B}\right]$ followed by $N_{B S} X M \mathrm{RF}$ precoder given by $F_{R}=\left[f_{1}^{R}, f_{2}^{R} \ldots \ldots f_{M}^{R}\right]$.The signal $x$ transmittedis represented by (1)

$$
x=F_{B} F_{R} S
$$

where $s=\left[s_{1}, s_{2} \ldots \ldots \ldots, s_{M}\right]$ is the M X 1 transmitted symbols vector, the covariance matrix is $E\left[s s^{*}\right]=\frac{\rho}{M} I_{M}$ where $\rho$ is the average total power transmitted.The total power constraint by normalizing $F_{B}$ that is $\left\|F_{R} F_{B}\right\|_{F}^{2}=M$.

$F_{R} \mathrm{RF}$ precoder elements are constrained to be constant magnitude phase factors whose entries are constant modulus satisfying $\left|\left[F_{R}\right]_{u, v}\right|^{2}=N_{B S}^{-1}$ and also assuming that the analog RF phase shifters are quantized angles with finite values as $\left[F_{R}\right]_{u, v}=\frac{1}{\sqrt{N_{B S}}} e^{j \emptyset_{u, v}}$, Where $\emptyset_{u, v}$ are the quantized angle.

By adopting for simplicity a narrow band block fading channel model given in [6],[16], the mth MS observes the signal received before combining as (2)

$$
r_{m}=H_{m} \sum_{n=1}^{M} F_{R} f_{n}^{B} s_{n}+n_{m}
$$

Where $H_{m}$ is the $N_{M S} X N_{B S}$ representing the mm-Wave channel matrix between the BS and the $m t h \mathrm{MS}$ and $n_{m}$ is the Gaussian noise $n_{m} \sim \mathcal{N}\left(0, \sigma^{2} I\right)$.

At the $m t h \mathrm{MS}$, the RF combiner $w_{m}$ processes the received signal $r_{m}$ as (3)

$$
y_{m}=w_{m}^{*} H_{m} \sum_{n=1}^{M} F_{R} f_{n}^{B} s_{n}+w_{m}^{*} n_{m}
$$

Where $w_{m}$ has the same constraints as that of RF precoderi.e the constant modulus and the quantized angles constraints.

The mm-wave Channel have limited scattering, so a virtual model is adopted which is a simplified model, thereby considering only azimuth angle $\theta$ i.e all the scattering is w.r.t horizontal beamforming only and neglecting the elevation angle $\varnothing$ as (4)

$$
H_{m}=\sqrt{\frac{N_{B S} N_{M S}}{L_{m}}} \sum_{l=1}^{L_{m}} \alpha_{m, l} b_{M S}\left(\theta_{R}^{m, l}\right) b_{B S}^{H}\left(\theta_{T}^{m, l}\right)
$$

Where $L_{m}=$ No. of multipath components

$b_{M S}\left(\theta_{R}^{m, l}, \emptyset_{R}^{m, l}\right), b_{B S}^{*}\left(\theta_{T}^{m, l}, \emptyset_{T}^{m, l}\right)$ are the beam steering vectors at the MS and the BS

$\theta$ is the Azimuth angle

$\varnothing$ is the Elevation angle

$\alpha_{m, l}$ is Complex channel gain

$\left(\theta_{R}^{m, l}, \emptyset_{R}^{m, l}\right),\left(\theta_{T}^{m, l}, \emptyset_{T}^{m, l}\right) \in[0,2 \pi]$ are the $l^{t h}$ path of angles of arrival/ departure (AOAs/AODs) respectively.If uniform linear array is assumed then

$$
b_{B S}(\varnothing)=\frac{1}{\sqrt{N_{B S}}}\left[1, e^{j \frac{2 \pi}{\lambda} d \sin (\varnothing)}, \ldots \ldots, e^{j\left(N_{B S}-1\right) \frac{2 \pi}{\lambda} d \sin (\varnothing)}\right]^{T}
$$

Where $\lambda$ is the wavelength of signal and $d$ is the distance between the antenna elements and in similar way the array response at the MS can also be presented.

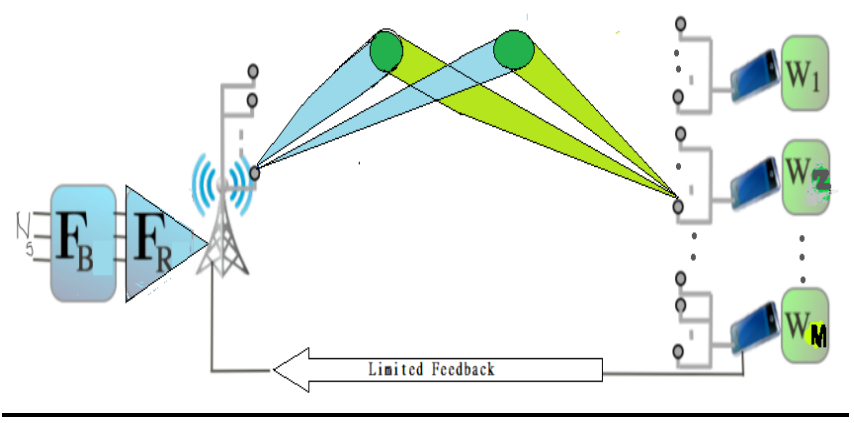

Fig1.Downlink system model where BS uses hybrid precoding and a large antenna array to serve multiple users and each MS has a limited feedback to the BS

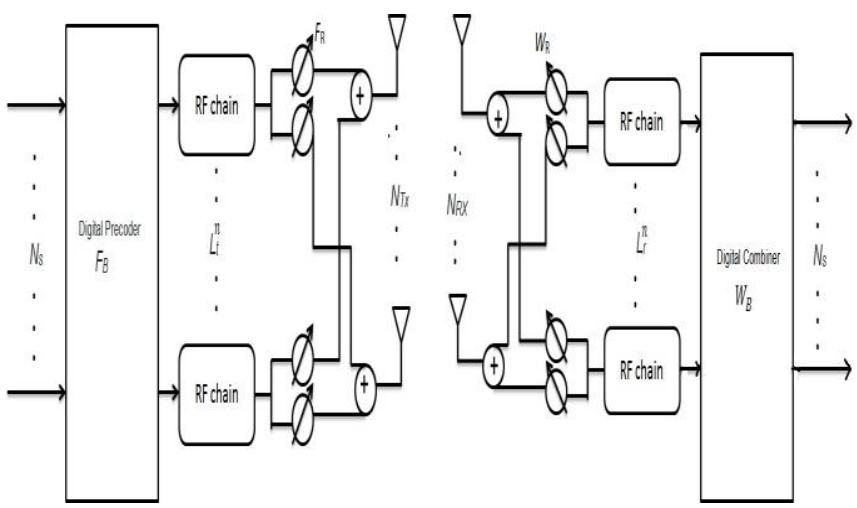

Fig.2.Block diagram of mm-Wave MIMO system with Hybrid precoding/combining

In section III , channel state information(CSI) $\mathrm{H}$ is assumed to be available at the Rx and is able to obtain $F_{o p t}$ but the knowledge of CSI may not be there in practical systems,so a limited feedback[17]-[19]is proposed to fulfill the requirements of channel information.Assume that $\mathrm{Rx}$ acquires $\mathrm{H}$ perfect knowledge ,calculates $F_{\text {opt }}$ and its hybrid $F=F_{R} F_{B}$ approximation and feedback information about the $F=F_{R} F_{B}$ back to the Tx as shown in Fig.1. 
The mm-Wave HPC problem formulation for a single user is described in section III below.

\section{MM-WAVEHYBRIDPRECODING/COMBINING( HPC) PROBLEM FORMULATION FOR A SINGLE USER}

Consider a mm-Wave system model after precoding and before combining given by (6)

$y_{N_{M S} X 1}=H x+n=H F_{R} F_{B} s+n=H F s+n$

After applying RF and BB combiners results in (7)-(8)

$$
\begin{aligned}
& \tilde{y}=W_{B}^{H} W_{R}^{H} y=W_{B}^{H} W_{R}^{H} H x+W_{B}^{H} W_{R}^{H} n \\
& \tilde{y}=W_{B}^{H} W_{R}^{H} H F_{R} F_{B} s+W_{B}^{H} W_{R}^{H} n=W H F s+W n
\end{aligned}
$$

Where Combiner $W=W_{R} W_{B} ; W_{B}^{H}=N_{S} X N_{R F}$

$$
W_{R}^{H}=N_{R F} X N_{M S}
$$

Let the noise covariance be $\sigma_{n}^{2} I$ that is $E\left\{n n^{H}\right\}=\sigma_{n}^{2} I$

At the output of combiner the noise covariance is given by

$$
R_{n}=\sigma_{n}^{2} W_{B}^{H} W_{R}^{H} W_{R} W_{B}
$$

Assume 's' comprises of zero-mean Gaussian symbols with covariance given by $E\left[s s^{H}\right]=\frac{1}{N_{S}} I$

The capacity of the channel is given by (11) using the equation (8)

$$
=\log _{2}\left|I+R_{n}^{-1} W_{B}^{H} W_{R}^{H} H F_{R} F_{B} F_{B}^{H} F_{R}^{H} H^{H} W_{R} W_{B}\right|
$$

Which is complicated and difficult to optimize.

Assume Rx can perform optimal maximum likelihood decoding,the mutual information achieved by Gaussian signaling is given by (12)

$$
I\left(F_{R}, F_{B}\right)=\log _{2}\left|I+\frac{1}{N_{s} \sigma_{n}^{2}} H F_{R} F_{B} F_{B}^{H} F_{R}^{H} H^{H}\right|
$$

The transmit precoding optimization problem can be formulated as (13)as $\left(F_{R}^{o p t} F_{B}^{o p t}\right)$

$$
=\underbrace{\operatorname{argmax}}_{\mathrm{F}_{\mathrm{R}}, \mathrm{F}_{\mathrm{B}}} \log _{2}\left|I+\frac{1}{N_{S} \sigma_{n}^{2}} H F_{R} F_{B} F_{B}^{H} F_{R}^{H} H^{H}\right|
$$

s.t $F_{R} \in \bar{F}_{R}$ set of all $N_{T} X N_{R F}$ matrices with unit magnitude elements.

Resolving the channel matrix $\mathrm{H}$ as the constituent elements of singular value decomposition (SVD) as given in (14)

$H=U \sum V^{H}$

Where $U=N_{R} X \operatorname{rank}(H)$ is receive combiner, values of SVD are in $\Sigma$ placed in descending order(strongest channel first) following water-filling algorithm and its size is given as

$$
\sum_{V^{H}=\operatorname{rank}(H) X N_{T} \text { is the transmit precoder }}=\operatorname{rank}(H) X \operatorname{rank}(H)
$$

Partition $\mathrm{V}$ and $\Sigma$ as given in (15)

$$
\Sigma=\left[\begin{array}{cc}
\Sigma_{1} & 0 \\
0 & \Sigma_{2}
\end{array}\right], \quad V^{H}=\left[\begin{array}{l}
V_{1}^{H} \\
V_{2}^{H}
\end{array}\right], \Sigma_{1}=N_{s} X N_{s}
$$

$V_{1}^{H}=N_{S} X N_{T}$ recall that the no. of transmitted symbols $\leq$ $\operatorname{rank}(\mathrm{H})$.

The mutual information can be apprioximated as (16)-(17)

$$
\begin{aligned}
& I\left(F_{R}, F_{B}\right)=\log _{2}\left|I_{N_{S}}+\frac{1}{N_{s} \sigma_{n}^{2}} H F_{R} F_{B} F_{B}^{H} F_{R}^{H} H^{H}\right| \\
& \approx \underbrace{\log _{2}\left|I_{N_{S}}+\frac{1}{N_{s} \sigma_{n}^{2}} \Sigma_{1}^{2}\right|}-(N_{s}-\underbrace{\|V_{1}^{H} \underbrace{F_{R} F_{B}}_{R}\|_{F}^{2}})
\end{aligned}
$$

Actual information loss in mutual information

Where $F_{R} F_{B} \approx V_{1}$ and the quantity $\left\|V_{1}^{H} F_{R} F_{B}\right\|_{F}^{2}$ should be equal to $N_{S}$ for second part of (17) to be zero .

For a conventional MIMO, the precoding is done at baseband digital precoder $F_{B}$ only,so the mutual information which is given in (17) can be rewritten as (18)-(19)

$$
\begin{aligned}
& =\log _{2}\left|I_{N_{S}}+\frac{1}{N_{s} \sigma_{n}^{2}} \Sigma_{1}^{2}\right|-\left(N_{S}-\left\|V_{1}^{H} F_{B}\right\|_{F}^{2}\right) \\
& =\log _{2}\left|I_{N_{S}}+\frac{1}{N_{S} \sigma_{n}^{2}} \Sigma_{1}^{2}\right| \text { when } F_{B}=V_{1}
\end{aligned}
$$

Thus, reduces to $F_{B}=V_{1}$, which is the optimal precoder for classical MIMO architecture.

To optimize the mutual information in hybrid precoder, $F_{R} F_{B}$ should be close to $V_{1}$ i.e $F_{R} F_{B} \approx V_{1}$. Hence the equivalent optimization problem is given by(20)

$$
\underbrace{\arg \min }_{\mathrm{F}_{\mathrm{R}}, \mathrm{F}_{\mathrm{B}}}\left\|V_{1}-F_{R} F_{B}\right\|_{F}^{2}
$$

Ideally $V_{1}=F_{R} F_{B}$ is desirable.

The model for channel matrix $\mathrm{H}$ for single-user case is(21)

$$
\begin{aligned}
& H_{m}=\sqrt{\frac{N_{B S} N_{M S}}{L}} \sum_{l=1}^{L} \alpha_{l} b_{M S}\left(\theta_{R}^{l}\right) b_{B S}^{H}\left(\theta_{T}^{l}\right) \\
& =\bar{B}_{M S}(\emptyset) H \bar{B}_{B S}(\emptyset)
\end{aligned}
$$

The transmit array response vectors form basis for row space of $H$ is $\bar{b}_{B S}\left(\theta_{T}^{l}\right)$ and the desired precoder is in (23)

$$
V_{1}=\bar{B}_{B S}\left(\emptyset_{T}\right) \bar{F}
$$

Ideally $\bar{B}_{B S}\left(\emptyset_{T}\right)$ can be made the RF precoder and $\bar{F}$ can be made the baseband precoder since the $\bar{B}_{B S}\left(\emptyset_{T}\right)$ entries are unit magnitude(its entries are not known in general) However, if $N_{R F}<L$, then one cannot obtain $V_{1}$ from $\bar{B}_{B S}\left(\emptyset_{T}\right)$. Since $\bar{B}_{B S}\left(\emptyset_{T}\right)$ has $L$ columns,one can linearly combine $N_{R F}$ columns of $\bar{B}_{B S}\left(\emptyset_{T}\right) . F_{R}$ can contain only $N_{R F}$ columns and $F_{B}$ can contain only $N_{R F}$ rows. The problem is modified by consideringan angular grid of size $G$ with $G \geq$ $N_{B S}$ or $N_{T}$.

The Dictionary is constructed as $B_{B S}\left(\emptyset_{T}\right)=\left[b_{B S}\left(\theta_{l}\right), 1 \leq\right.$ $l \leq G] \quad, \quad$ and the resulting optimization problem is given (24) 


\section{Hybrid Precoding/Combining for single-user and Multi-Usersin mm-Wave MIMO Systems}

$\arg \min \|V_{1}-\underbrace{B_{B S}\left(\emptyset_{T}\right)} \tilde{F}_{B}\|_{F}^{2}$

s.t $\left\|\operatorname{diag}\left(\widetilde{F}_{B} \tilde{F}_{B}{ }^{H}\right)\right\|_{0}=N_{R F}$ : Enforces sparsity

and $\left\|B_{B S}\left(\emptyset_{T}\right) \tilde{F}_{B}\right\|_{F}^{2}=N_{S}$

$F_{B}$ has to be a block sparse matrix, angles are unknown , create a dictionary and choose among them the best vector in dictionary which approximates the $V_{1}$. Finally,extract the non-zero rows from $\tilde{F}_{B}$ for baseband precoder $F_{B}$ and corresponding columns from matrix $B_{B S}\left(\emptyset_{T}\right)$ for RF precoder $F_{R}$ as shown in Algorithm below.

In the OMP algorithm, the AOA/AOD space [0 to $\pi]$ is partitioned into $G$ grids. A dictionary corresponding to array response vectors of all the possible angles of arrivals/departures is created. Dictionary size depends on individual resolution where all the received array response vectors corresponding to a specific resolution may be placed.

The angle of arrival at Rx is not known but it will be close to one of the angle of arrivals present in dictionary. If the particular AOA/AOD is not present in the dictionary then it's taken as zero. In a mm-wave system, multipath dominant components are very few, the problem is solved on path by path basis which is an efficient way of doing estimation for better accuracy. Only those entries of the matrix will be non-zero, if you have that corresponding angle of arrival and departure active. The Matrix with large number of zeros and only few non-zeros, is a sparse Matrix. The columns contains the angle of arrival/departure pairs. The beam space matrices are similar to DFT matrices. The Algorithm for mm-Wave MIMOhybrid precoder/combiner using SOMP is described below:

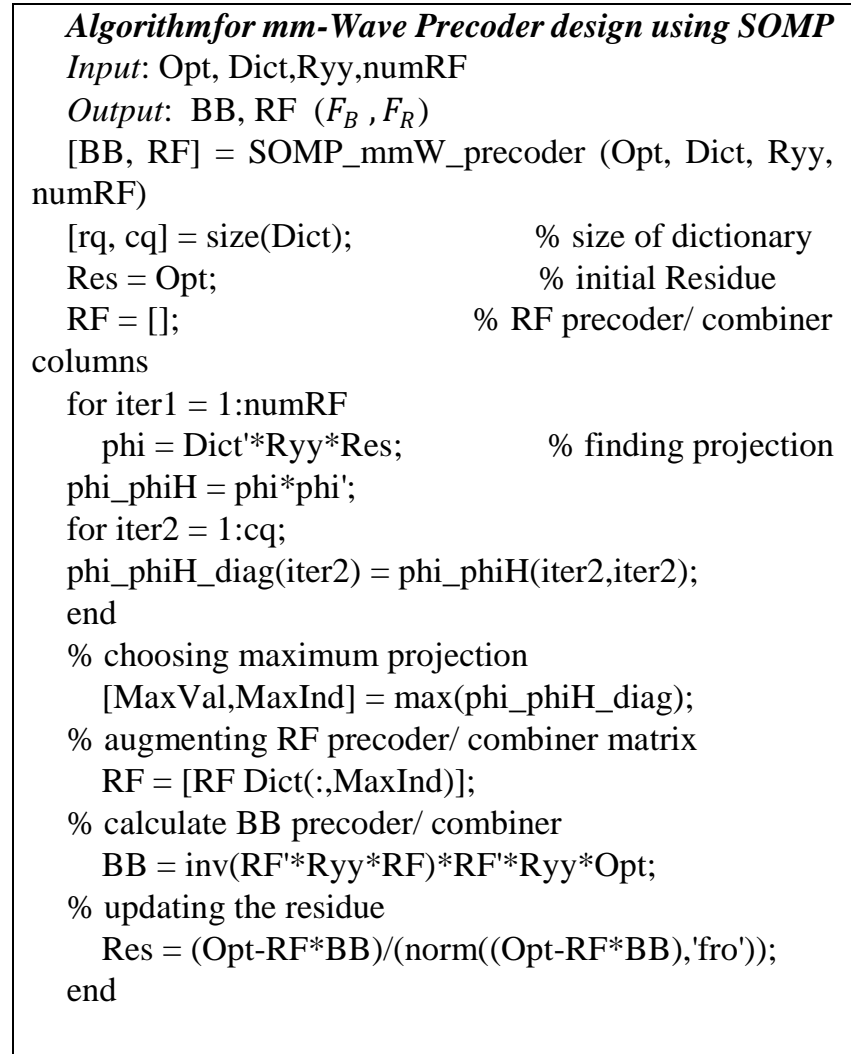

The HP is finally obtained as $F_{B}=\frac{\sqrt{N_{S}} F_{B}}{\left\|F_{R} F_{B}\right\|_{F}}$ and the SOMP algorithm returns $F_{R}, F_{B}$ both the precoders. The algorithm doesn't assume that directional cosine vectors to be known and there will be an approximation error always when $N_{R F}<L$.

The analog combiner problem occurs in the same manner as analog Precoder problem and to achieve the ideal analog combiner by replacing the symbols $F_{R}$ and $\mathrm{H}$ by $W_{R}$ and $H_{1}$ where $H_{1}=H F_{R} F_{B}$, the above algorithm can be applied directly by using minimum mean square error (MMSE) combining.

\section{MM-WAVE HPC PROBLEM FORMULATION FOR MULTI- USERS}

To efficiently design the analog RF and digital baseband precoders at the BS and also analog combiners at the MS's , the sum-rate of the system is maximized.The signal received at the $m^{\text {th }}$ MS in equation (2) is further processed by the RF combiner $w_{m}$ and the rate achieved by the user $m$ is given (25)

$$
R_{m}=\log _{2}\left(1+\frac{\frac{\rho}{M}\left|w_{m}^{*} H_{m} F_{R} f_{m}^{B}\right|^{2}}{\frac{\rho}{M} \sum_{n \neq m}\left|w_{m}^{*} H_{m} F_{R} f_{m}^{B}\right|^{2}+\sigma^{2}}\right)
$$

The system sum rate is given by (26)-(27)

$$
R_{\text {sumrate }}=\sum_{m=1}^{M} R_{m}
$$

If the performance metric is the sumrate then the precoding problem is to find $F_{R}^{*},\left\{f_{m}^{* B}\right\}_{m=1}^{M},\left\{w_{m}^{*}\right\}_{m=1}^{M}$ that simplifies to

$$
=\arg \max \sum_{m=1}^{M} \log _{2}\left(1+\frac{\frac{\rho}{M}\left|w_{m}^{*} H_{m} F_{R} f_{m}^{B}\right|^{2}}{\frac{\rho}{M} \sum_{n \neq m}\left|w_{m}^{*} H_{m} F_{R} f_{m}^{B}\right|^{2}+\sigma^{2}}\right)(27)
$$

\section{SIMULATION RESULTS}

To simulate results, MATLAB R2019a simulator is used. Consider the $\mathrm{Tx}$ and $\mathrm{Rx}$ consisting of a uniform linear array with $N_{T_{X}}=N_{R_{X}}=32, N_{R F}=6$ which is set equal to $N_{S}$ and $\mathrm{L}$ is the sparsity level which is set to 8.The AOA/AOD space are divided into $G=64$ grids and a dictionary is created mapping to array response vectors of all the possible angles of arrivals/departures.

The results shows that the capacity of OMP based hybrid precoderapproaching Conventional MIMO whose results are illustrated in figure 3 .

The procedure for Hybrid Precoder design in mm-Wave MIMO system using SOMP is presented below:-

Step 1:

Set up the required parameters for mm-Wave hybrid precoder design as shown in Table-1 below:

Step 2:

Initialize the Grid quantized transmit/receive array beam response vectors and generate their dictionary matrix. Here the $\cos \theta$ values are directly generated not the angles which is given by $\operatorname{dirCos}=2 / \mathrm{G}^{*}(\mathrm{I}-1)-1$, for $\mathrm{I}=1: \mathrm{G}$, for $\mathrm{K}=1: \mathrm{Nt}$

B_T $(\mathrm{K}, \mathrm{I})=1 / \operatorname{sqrt}(\mathrm{t}) * \exp (-\mathrm{j} * \mathrm{pi} *(\mathrm{~K}-1) * \operatorname{dirCos})$

B_R=B_T;for simplicity 
Stesp 3:

Generate Channel gain and Channel matrix and compute singular value decomposition (SVD) of $\mathrm{H}$

$\operatorname{chGain}=\frac{1}{\sqrt{2}}((\operatorname{randn}(\mathrm{L}, 1)+\mathrm{j} * \operatorname{randn}(\mathrm{L}, 1))$;

$\mathrm{H}=\sqrt{\frac{N t * N r}{L}} * \mathrm{~B} \_\mathrm{R} \_$genie $* \operatorname{diag}($ chGain $) * \mathrm{~B} \_\mathrm{T} \_$genie';

$[\mathrm{U}, \mathrm{S}, \mathrm{V}]=\operatorname{svd}(\mathrm{H})$;

Table I: Set up Simulation parameters

\begin{tabular}{|l|l|}
\hline \multicolumn{2}{|c|}{ Simulation Parameters } \\
\hline \multicolumn{1}{|c|}{$N_{T_{X}}$} & 32 \\
\hline \multicolumn{1}{|c|}{$N_{R_{X}}$} & 32 \\
\hline RF chains & 6 \\
\hline N_Beam & 24 \\
\hline Grid $G$ & 64 \\
\hline ITER & 100 \\
\hline L sparsity & 8 \\
\hline dirCos & $(-1,1]$ \\
\hline SNRdB & $-5: 5: 10$ \\
\hline
\end{tabular}

Step 4:

Obtain optimal unconstraint precoderFopt $=\mathrm{V}(:, 1: \mathrm{Ns})$

Step 5:

OMP based Hybrid precoder design is implemented

[FBB,FRF]=SOMP_mmW_precoder(Fopt,B_T,eye(t),nu $\mathrm{mRF}$ )

FBB_NORM=sqrt(Ns)/(norm(FRF*FBB,'fro' $)) * F B B$

Step 6:

Determine the Capacity of optimal unconstraint MIMO precoder/combiner .

C_MIMO(i_snr)=C_MIMO(i_snr)+mimo_capacity $(\mathrm{Wm}$ mse_opt'*H*Fopt, $1 / \mathrm{Ns} *$ eye(Ns),np*Wmmse_opt'*Wmmse opt);

Step 7:

OMP based receive hybrid combiner design and the capacity of OMP-based precoder/combiner

[WBB,WRF]=SOMP_mmW_precoder $\left(\mathrm{Wmmse} \_\right.$Hyb,B_ R,Ryy,numRF);

C_HYB(i_snr) $=$ C_HYB(i_snr $)+$ mimo_capacity $\left(\mathrm{WBB}^{\prime} * \mathrm{WR}\right.$ $\mathrm{F}^{*} \mathrm{H}^{*} \mathrm{FRF} * \mathrm{FBB} \_\mathrm{NORM}, 1 / \mathrm{Ns} *$ eye(Ns),np*WBB'*WRF'* WRF*WBB);

C_MIMO=C_MIMO/ITER;C_HYB=C_HYB/ITER;

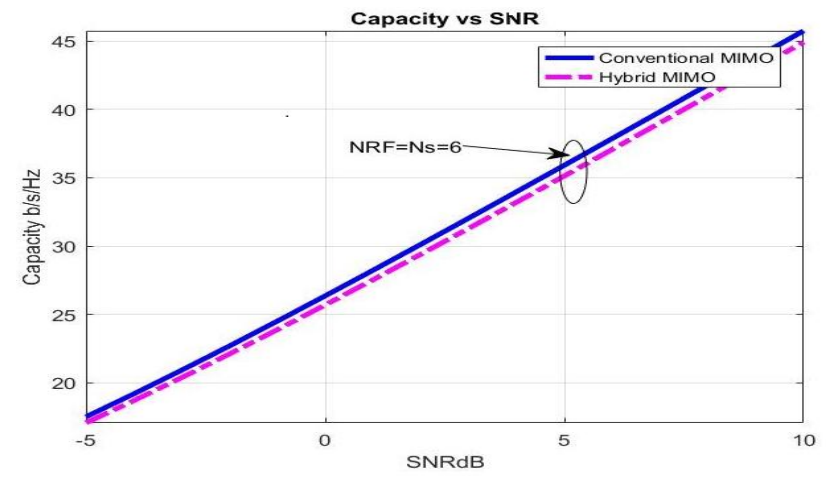

Fig.3.Capacity vs SNR for conventional MIMO
withHybrid MIMO using OMP estimatorfor $\mathrm{NRF}=\mathrm{Ns}=6$

In Fig. 3, the parameters are set up as mentioned in the Table 1, the performance analysis of conventional MIMO is compared with the Hybrid MIMO using OMP algorithm. It portray's that the performance loss is due to assumption of the quantized angle of arrival and departures in dictionary which is very small in OMP algorithm and the capacity approaches the conventional performance.OMP algorithm performs the computation with very few number of samples efficiently, shows an advantage over conventional MIMO estimators.

In Fig.4 spectral efficiency (SE) obtained in a $64 \times 16$ with a planar array at both $\mathrm{Tx}$ and $\mathrm{Rx}, N_{R F}=4, N_{s}=$ 1 or 2 streams and over 500 channel realization. The demonstrated results potrays that the proposed OMP based sparse precoding and combining is accurately approximating the optimal unconstraint solution in comparision to conventional analog beamsteeringi.eat $\mathrm{SNR}=0 \mathrm{~dB}$,it is observed that SE is lower by $7 \mathrm{~b} / \mathrm{s} / \mathrm{Hz}$ in analog beemsteeringwhen compared to optimal and sparse precoding/combing which is close to $15 \mathrm{~b} / \mathrm{s} / \mathrm{hz}$ and the performance gap is increasing as SNR varies from $-20 \mathrm{~dB}$ to $20 \mathrm{~dB}$ as observed in Fig.4.

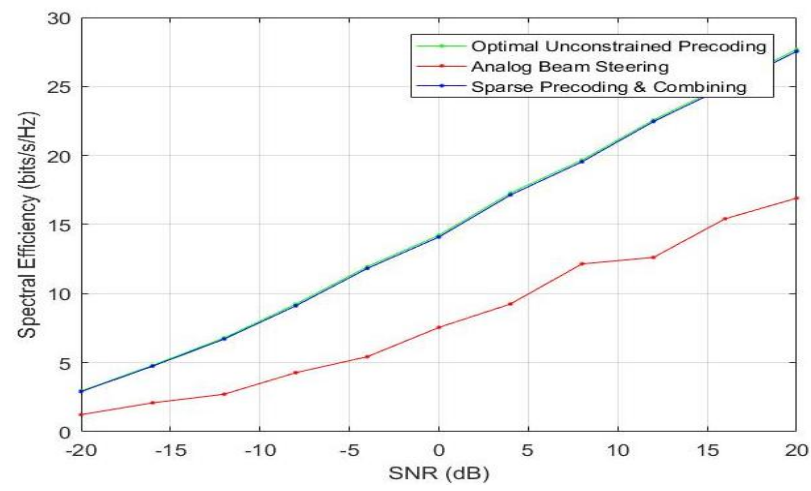

Fig.4.SE vs SNRfor $64 \times 16 \mathrm{~mm}$-Wave $\operatorname{system} N_{R F}=$ $4, N_{s}=1$ or 2 streams (single user)

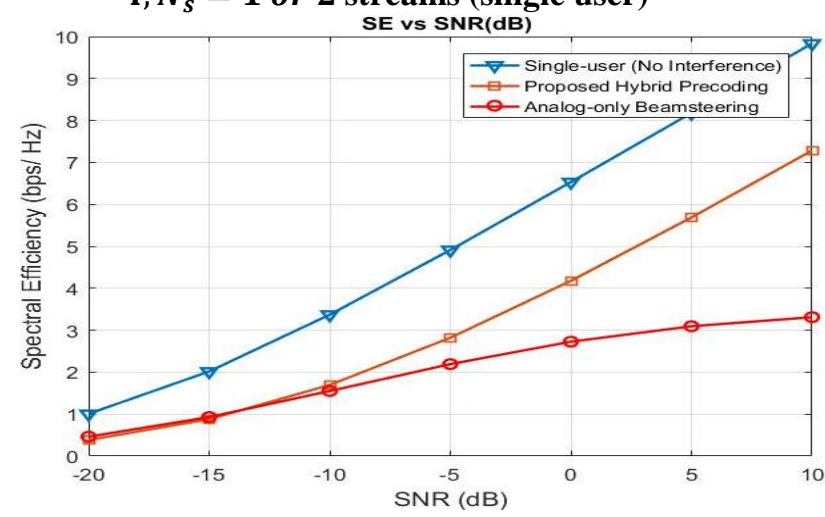

Fig.5.SEvsSNR(dB) using HP and beamsteering algorithm with channel knowledge(Multi-users=4)

In Fig. 5, Consider the system model in section II where a BS employs 8 x 8 UPA with four MS's, the AOA/AOD are distributed uniformly in $\left[-\frac{\pi}{2}, \frac{\pi}{2}\right]$ is assumed. The performance achieved by HP is compared with SU rate and with beamsteering. The results illustrates that the performance of $\mathrm{HP}$ is close to $\mathrm{SU}$ (without interference) performance, which is due tothe cancellation of residual (MUI)multi-user interference. 


\section{Hybrid Precoding/Combining for single-user and Multi-Usersin mm-Wave MIMO Systems}

The Fig.5 also illustrates the HP gain in SE over AB which increases with SNR as analog performance rate is limited with interference.

\section{CONCLUSION}

A Comparative analysis for HPC for mm-Wave MIMO system with analog beamsteering and optimal digital precoding for single user and multi-user have been presented using OMP algorithm. The MATLAB simulations illustrates the outperformance of OMP algorithm which gives high capacity compared to ABand approaches the optimal digital precoding.The objective of this paper was to analyse and improve hybrid precoding/combining so that it can be incorporated to improve spectral efficiency and capacity in mm-Wave MIMO system for sparse channel estimation.

Future extension will be to use the Artificial Intelligence(AI)based learning algorithms to enhance hybrid precoding/combining performance further to efficiently estimate sparse channels in mm-Wave MIMO systems.

\section{REFERENCES:}

1. S. K. Yong et.al "An overview of multigigabit wireless through millimeter wave technology: Potentials and technical challenges," EURASIP Journal on Wireless Communications and Networking, vol. 2007, no. 1, p. 078907, 2006.

2. R. W. Heath, N. Gonzalez-Prelcic,et.al "An overview of signal processing techniques for millimeter wave MIMO systems," IEEE Journal of Selected Topics in Signal Processing, vol. 10, no. 3, pp 436-453, 2016.

3. F. Giannetti et.al, "Mobile and personal communications in the $60 \mathrm{GHz}$ band: A survey," Wireless Personal Communications, vol. 10, no. 2, pp. 207-243, 1999.

4. S.Kutty et.al, "Beamforming for millimeter wave communications: An inclusive survey, "IEEECommun.SurveysTuts.,vol.18,no.2,pp.949973, Second Quarter 2016.

5. Z. Pi et.al, "An introduction to millimeter-wave mobile broadband systems," IEEE Commun. Mag., vol. 49, no. 6, pp. 101-107, 2011

6. El Ayach, et al., Spatially sparse precoding in millimeter wave MIMO systems. IEEE transactions on wireless communications, 2014. 13(3): p. $1499-1513$.

7. J. Wang, et al., "Beam codebook based beamforming protocol for multi-Gbps millimeter-wave WPAN systems," IEEE Jour. Select. Areas in Commun., vol. 27, no. 8, pp. 1390-1399, Nov. 2009.

8. S. Hur, et al.,"Millimeter wave beamforming for wireless backhaul and access in small cell networks," IEEE Trans. Commun., vol. 61, no. 10 , pp. 4391-4403, Oct. 2013.

9. Y. Tsang, et al., "Coding the beams: Improving beamforming training in mmwave communication system," in Proc. Glob. Telecom. Conf., Houston, TX, Dec. 2011, pp. 1-6.

10. A. Sayeed et.al, "Maximizing MIMO capacity in sparse multipath with reconfigurable antenna arrays," IEEE Journal of Selected Topics in Signal Processing, vol. 1, no. 1, pp. 156-166, June 2007.

11. J. Brady, et.al "Beamspace MIMO for millimeterwave communications: System architecture, modeling, analysis, and measurements," IEEE Trans. on Ant. and Propag., vol. 61, no. 7, pp. 3814-3827, July 2013.

12. "IEEE 802.11ad standard draft D0.1." [Online]. Available: www.ieee802.org/11/Reports/tgad update.htm

13. T. Baykas, et.al, "IEEE 802.15.3c: the first IEEE wireless standard for data rates over $1 \mathrm{~Gb} / \mathrm{s}$," IEEE Comm. Mag., vol. 49, no. 7, pp. 114-121, July 2011.

14. C. Kim, et.al, "Multi-beam transmission diversity with hybrid beamforming for MIMO-OFDM systems," in Proc. of IEEE Globecom Workshops, Atlanta, GA, Dec. 2013, pp. 61-65.

15. A. Alkhateeb et.al, "Hybrid precoding for millimeter wave cellular systems with partial channel knowledge," in Proc. of Info. Th. and App. Workshop, Feb 2013, pp. 1-5.

16. Nazeerunnisa,MadhaviTatineni, "Sparse CE in mm-Wave Hybrid MIMO systems" IJITEE ISSN:2278-3075

17. D. Love et.al, "Limited feedback unitary precoding for spatial multiplexing systems," IEEE Trans. Inf. Theory, vol. 51, no. 8, pp. 2967-2976, Aug. 2005.
18. D. Love, R. W. Heath, et.al, "Grassmannian beamforming for multiple-input multiple-output wireless systems," IEEE Trans. Inf. Theory, vol. 49, no. 10, pp. 2735-2747, Oct. 2003.

19. J. C. Roh et.al, "Design and analysis of MIMO spatial multiplexing systems with quantized feedback," IEEE Trans. Signal Process., vol. 54, no. 8, pp. 2874-2886, 2006.

\section{AUTHORS PROFILE}

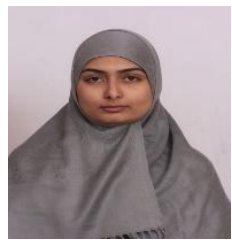

Nazeerunnisa is working as a Sr.Assistant Professor in the Department of ECE at MuffakhamJah college of engineering and technology, Hyderabad, India. She is currently pursuing Ph.D from GITAM University, Vishakhapatnam, India. She received her M.Tech in Systems and Signal Processing at JNTU Hyderabad in 2011. She has over 16 years of Teaching experience. Her main research interest includes channel Estimation in wireless communications systems, Digital Signal Processing, Sparse signal processing for mmWave MIMO systems.

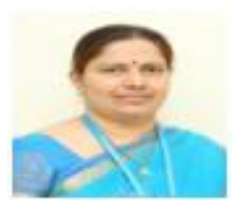

Dr. T. Madhavi is currently working as Professor in the Department of EECE, GITAM, Visakhapatnam, India. She received her M.Tech in Radar and Microwave Engineering in 2004 and Ph.D in 2013 from Andhra University, India. She has over 25 years of experience in Industry and Teaching. She has published several papers in Reputed Journals like IET, Springer, etc. Her main research interest includes modeling and performance analysis of wireless communications systems, Cellular/mobile communications and Wireless Sensor Networks. 